\title{
Reciprocal Conditions for Spatial Mirror Inversed Sentences in Modern Chinese
}

\author{
Xiaolan Gan* \\ School of Foreign Languages, Sichuan University of Science and Engineering, China
}

*Corresponding Author: Xiaolan Gan, School of Foreign Languages, Sichuan University of Science and Engineering, China

\begin{abstract}
Mirror inversed sentences (MISs) is a special syntactic-semantic phenomenon in modern Chinese with the typical characteristics of "reciprocal syntactic components and unchanged propositional meaning". At present, whether discussing the reciprocal conditions of MISs from the perspective of predicates, body words and constructions, or from a comprehensive point of view, they are all faced with a common problem: they can never answer the question of why the propositional meaning of MISs remains unchanged. This paper summarizes the common conditions for realizing the interchangeability of body words in spatial MISs, and explores the deep cognitive reasons for the interchangeability. This paper finds that the existence of "symmetry" semantic relations in the spatial dimension is the common necessary condition for this kind of MISs to realize the interchangeability of body words. In other words, the conceptual semantic symmetry of MISs is the premise of its formal symmetry, and formal symmetry is the reflection of its conceptual semantic symmetry.
\end{abstract}

Key terms: MISs; Spatial relations; Symmetric semantic relation

\section{INTRODUCTION}

Chinese word order is relatively fixed. "X" and " $Y$ " (body words) before and after "P" (predicates) in sentence pattern " $\mathrm{X}+\mathbf{P}+\mathrm{Y}$ " can't be exchanged arbitrarily. When the body words are reciprocal, the propositional meaning often changes or is no longer reasonable. However, there are some special phenomena that the propositional meaning does not change after the reciprocity. For example, "Xichang tong tielu $\leftrightarrow$ Tielu tong Xichang (Xichang connects the railway $\leftrightarrow$ The railway connects Xichang)". This kind of sentence pattern has the typical characteristics of "reciprocity of syntactic components and unchanged propositional meaning" and is thus named as "mirror inversed inversion (MISs)" and divided into six categories: attributive, spatial, temporal, attributive-temporal, spatialtemporal and attributive-spatial-temporal. (Gan, 2019)

Why can the body words in MISs carry out reciprocity around predicates while keeping the propositional meaning basically unchanged? Most of the existing researches make descriptive analysis of the internal factors of language (i.e. predicates and body words) but lack the analysis adequacy and explanation adequacy. Although some researchers began to pay attention to revealing the motivation of the interchangeability of body words from the external factors of language (i.e. constructions), these explanations are mostly one-sided, and often only aim at some subclasses. In view of the fact that events have three basic elements of attribute, space and time (Kant, 2004), this paper attempts to discuss the reciprocal conditions for body words in spatial MISs from both positive and negative aspects and explores the deep cognitive reasons for the interchangeability of body words.

\section{Previous Studies on the Reciprocal Conditions of Miss}

\subsection{Studies from the Perspective of Predicates}

Researchers notice the double-faced features of verbs (verb as the major component of predicates) on the interchangeability of MISs early. Ding et al. (1961) mentions that "Yige da bing jia yigen youtiao (a big cake is filled with a deep-fried dough stick)" and "Yigen youtiao jia yige da bing (a deep-fried dough stick is filled with a big cake)" can be grammatical only if the verb "jia (be filled with)" has double faces. Ma (1955) agrees and formally puts forward the definition of "double-faced verbs", while Bai (1994) further discusses their classification in detail. 
Later researchers focus more on the semantic requirements of verbs in MISs. Zhu (1981) points out that "Zai heiban shang xie zi (On the blackboard are written the characters)" and "Zi xie zai heiban shang (The characters are written on the blackboarde)" can change each other because the verb "xie (write)" contains the meaning of "attaching" to "something". Chen (1986) states that there were transformational relationships between the body words of the sentences with the verbs containing the senses of "allow". For example, "Zhetiao lu zou qiche (This way is allowed to go by car)" can be said to be "Qiche zou zhetiao lu (The car is allowed to go on this way)". Similarly, Lu (1993) also believes that verbs with the meanings of "enough and permission" are the conditions for "Shige ren chi yiguo fan (Ten people eat a pot of rice)" to be derived from "Yiguo fan chi shige ren (*A pot of rice eat ten people)".

Different from the above emphasis on "verbs", some researchers think that we should pay more attention to the "verb complement (the other component of predicates)". The importance of "verbs" and "their complements" should be reversed, that is, "complements" should be considered to be more important than "verbs" (Li, 1984). Zhan (1989) agrees and puts forward that "man (be filled with)" in the sentence "Si kuang tian man le hong liu lv qin (Four boxes are filled with red willows and green birds)" can be used to both describe the two body words. Wang (1992) also points out that the complement "si (much)" after the verb "xiang (miss)" in "Wo xiang si ni (* I miss much you)" either points toward "wo" or "ni". Lu \& Zhang (2013) also finds that "si (much)" plays a key role in the inversion of the generalized verb-resultative construction.

Ren (2001), however, finds that there are some examples that are difficult to explain only from the single perspective of verbs or complements. He points out that the "verbs and its complements" are equally important in the reciprocity of MISs. For example, in the sentence "Lao Wang he zui le jiu (Lao Wang is drunk with wine)", the structural center of the predicate "he zui le (is drunk) " is the verb " he (drink)". In the sentence "Jiu he zui le Lao Wang (* Wine is drunken with Lao Wang)", the center shifts to the result complement "drunk". However, Ren cannot explain why "Wo ting dong le zheju hua (I understood this sentence)" can be said, but "*Zheju hua ting dong le wo (*this sentence understood me)" cannot be said.

Exploring the reciprocal conditions of MISs from the perspective of predicates (verbs and their complements) can explain the reasons for the interchangeability of body words in some sentence patterns, but most of them are beyond the scope of discussion. Therefore, many scholars try to examine the reciprocity conditions from the perspective of the body words.

\subsection{Studies from the Perspective of Body Words}

Lv (1987) firstly notices that the semantics of body words had an important influence on the grammatical format of the sentences. When discussing "Shui shi Zhang Laosan (Who is Zhang Laosan)" and "Zhang Laosan shi shui (Zhang Laosan is who)", he puts forward that "shui (who)" has two functions: "shui" in the former sentence refers to "the person"; while "shui" in the latter is used for "explanation". However, not all the body words have different functions, such as the body words "a candle" and "a dime" in "Yizhi lazhu yimao qian (A candle costs a dime) $\leftrightarrow$ Yimao qian yizhi lazhu (A dime costs a candle)" both express the quantity of goods and the quantity of prices.

More scholars noticed that the semantic roles of body words in the reciprocity of MISs. Chen (1994), based on Dowty's prototype role theory (1991), puts forward the semantic role priority sequence of the body words, namely: agent $>$ sentiment $>$ tool $>$ being $>$ location $>$ theme $>$ patient. Based on this sequence, Chen finds that the reciprocity conditions for MISs are: "semantic roles close to the middle of the sequence" and "semantic roles not at the two ends of the sequence, but close to each other". For example, the reason why "Zhuantou dian le qiang (a)" can be changed into "Qiang dian le zhuantou (b)" is that the role of the body word "Zhuantou" in sentence (a) is "tool" and the role of the body word "Qiang" is "location". Their semantic roles are close to the middle of the sequence. However, the above sequence cannot explain the "inversion of agent and patient", such as "Bage ren chi zheguo fan (eight people eat this pot of rice)" and "Zheguo fan chi bage ren (This pot of rice eat eight people)".

Her (2009) tries to explain the "inversion of agent and patient" by adding the concept of compound semantic roles and the semantic role priority sequence "agent $>$ beneficiary $>$ goal/experience $>$ tool $>$ patient/object $>$ location/amplitude" proposed by Huang (1993). Her assumes that some body words 
can acquire two semantic roles at the same time. For example, in the MISs "Shige ren chi yiguo fan (Ten people eat a pot of rice) $\leftrightarrow$ Yiguo fan chi shige ren (A pot of rice eat ten people)", the body word "Shige ren (Ten People)" can get a compound semantic role of "agent- amplitude", while the other body word "yiguo fan (a pot of rice)" can only get the role of "object". In "Shige ren chi yiguo fan", the role "agent" of "shige ren" is highlighted, they are in front of "patient" in the priority sequence and get the subject position; However, in the case of "Yiguo fan chi shige ren", the role "amplitude" of "ten people" is highlighted, and it is behind the "patient" in the priority sequence to obtain the object position. From the perspective of highlighting the composite semantic role, Her explains the "inversion of agent and patient", but fails to further explore where the composite roles are from.

Li (2010) argued that Her (2009) faces one problem: the subject or the object cannot acquire a single role while the other acquires a compound role. He points out that the examples of "Shige ren chi yiguo fan" and "Yiguo fan chi shige ren" were not really inversion of agent and patient, because the meanings of "Chi (eat)" in the two sentences are different. Specifically, "chi" in "Shige ren chi yiguo fan" can mean both "event" and "relationship". "chi" in "Yiguo fan chi shige ren", however, can only mean "relationship". The problem lying in Li (2010) is that two different explanations for verbs are bound to cause redundancy in the meaning of the verbs. Furthermore, like Her (2009), Li does not point out the specific conditions for obtaining semantic roles. In addition, what is "relationship"? Where does this "relationship" come from? He also does not explain.

Starting from the body words, this perspective can explain the reasons for the reciprocal of body words in some sentence patterns to a certain extent, but most of them are still beyond the scope of its discussion.

\subsection{Studies from the Perspective of the Constructions}

The discussion from this perspective mainly focuses on the "supply-verb" sentence patterns. Lu (2004) clearly points out that the reason why the body words of "Yiguo fan chi sanshige ren (One pot of rice for 30 people)" and "Sanshige ren chi yiguo fan (30 people for one pot of rice)" can realize reciprocity lies in the fact that such sentence patterns express the construction meaning of "accommodation". Ding (2006) further attributes the framework of "accommodation" to the basic cognitive framework of "container-content".

In addition, Yue (2009) also believes that one of the deep reasons for the reciprocity of body words is the independent construction meaning when discussing the pattern " $\mathrm{S}+\mathrm{W}+\mathrm{Si}$ (death) + O". Its construction meaning is that they all represent subjective feelings of polarity.

To a certain extent, the study of reciprocal conditions of MISs from the perspective of constructions has increased the intensity of the study, but it is mainly carried out in a small range such as "supplyverb" sentences. Moreover, the discussion cannot answer such questions: if the meaning of a sentence comes from the construction, then where does this "construction" come from?

\subsection{Summary}

In a word, at present, no matter from the perspective of predicates, body words or constructions, all the previous studies are facing a common problem: they have never been able to answer the question of why the "propositional meaning" of MISs remains unchanged, that is, they have not touched on the deep root of the reciprocal of body words in this kind of sentence patterns.

\section{SPATial RELATiONSHiP}

Spatial relations involve the relations among four geometric objects: points, lines, surfaces and bodies. Among them, points can be used to represent the positions of points in space, such as nails, trees and telegraph poles. Lines can be used to represent linear objects in three-dimensional space, such as railways, highways, bridges, rivers, power transmission lines, etc. Surfaces can be used to represent surface objects in three-dimensional space, such as playgrounds, lakes, forest coverage areas, etc. Bodies can be used to represent bodies in three-dimensional space, such as people, cars, houses, etc. According to the combinational forms of various geometric objects, the spatial relations can be logically summarized into the following 10 types: point-point relations, point-line relations, point-surface relations, point-body relations, line-line relation, line-surface relation, line-body relation, surface-surface relation, surface-body relation, body-body relation. In general, the 
description of the above 10 spatial relationships can be carried out from three angles of distance, direction and topology.

The spatial distance relation describes the relationship between geometric objects by measuring the distance in the space, which reflects the geometric proximity between geometric objects, such as "the school is 5 kilometers away from home" (in this sentence, the distance between the school and home is measured).

The spatial direction relation describes the azimuth relation of one geometric object relative to another geometric object, which is uniquely determined by three elements: the target object, the reference object and the fixed reference point (often referred to as the North Pole). Frequently used directional relationships include: east, south, west and north, such as "Hainan Island is in the south of China" (in this sentence, the direction of Hainan is determined by China). There is also a directional relationship called positional directional relationship: up, down, front, back, left and right, etc., such as "books are on the table" (in this sentence, the direction of books is determined by the table).

Spatial topological relation is the invariance of topological properties under topological transformation, such as the adjacency and connectivity of spatial targets. The spatial topological relation is usually determined by the target, reference and topological predicates, such as "Wuhan is close to the Yangtze River" (in this sentence, the topological relation between Wuhan and the Yangtze River is described) and "Xichang is connected to the railway" (in this sentence, the topological relation between Xichang and the railway is described).

The following is a quadruple model to describe the topological relationship between geometric objects. The quadruple model regards the spatial target as a point set, and uses the boundary between the two point sets and the intersection between the interior to form the following quadruple:

$T_{\text {Top }}(X, Y)=\left(\begin{array}{ll}L_{X} \cap L_{Y} & L_{X} \cap Y^{0} \\ X^{0} \cap L_{Y} & X^{0} \cap Y^{0}\end{array}\right)$

In formula (1), $\mathrm{L}_{\mathrm{X}}$ and $\mathrm{X}^{0}$ are the boundaries and interior of target $\mathrm{X}$, and $\mathrm{L}_{Y}$ and $\mathrm{Y}^{0}$ are the boundaries and interior of target $Y$. In pure mathematics, the value of each term of the quaternion can be empty or non-empty. The quaternion has 16 possible topological relations, excluding 8 that cannot be established, and the remaining 8 are shown in Table 1:

Table1. Common spatial topological relations between geometric objects

\begin{tabular}{|c|c|c|c|c|}
\hline Matrix & Valency & Surface-surface & semantics & relations \\
\hline$\left(\begin{array}{cc}\neg \varnothing & \varnothing \\
\varnothing & \varnothing\end{array}\right)$ & $\begin{array}{l}\text { There is an intersection between the } \\
\text { boundaries of } \mathrm{X} \text { and } \mathrm{Y} \text {; There is no } \\
\text { intersection between the boundary of } \\
\mathrm{X} \text { and the interior of } \mathrm{Y} \text {; There is no } \\
\text { intersection between the interior of } \mathrm{X} \\
\text { and the boundary of Y; There is no } \\
\text { intersection between the interiors of } \\
\mathrm{X} \text { and Y. }\end{array}$ & & $\begin{array}{l}X \text { and } Y \text { are } \\
\text { connected }\end{array}$ & Connection \\
\hline$\left(\begin{array}{cc}\neg \varnothing & \neg \varnothing \\
\neg \varnothing & \neg \varnothing\end{array}\right)$ & $\begin{array}{l}\text { There is an intersection between the } \\
\text { boundaries of } \mathrm{X} \text { and } \mathrm{Y} \text {; There is an } \\
\text { intersection between the boundary of } \\
\mathrm{X} \text { and the interior of } \mathrm{Y} \text {; There is an } \\
\text { intersection between the interior of } \mathrm{X} \\
\text { and the boundary of } \mathrm{Y} \text {; There is an } \\
\text { intersection between the interiors of } \\
\mathrm{X} \text { and Y. }\end{array}$ & & $\begin{array}{l}X \text { and } Y \text { are } \\
\text { intersected. }\end{array}$ & Intersection \\
\hline$\left(\begin{array}{cc}\neg \varnothing & \varnothing \\
\varnothing & \neg \varnothing\end{array}\right)$ & $\begin{array}{l}\text { There is an intersection between the } \\
\text { boundaries of } \mathrm{X} \text { and } \mathrm{Y} \text {; There is no } \\
\text { intersection between the boundary of } \\
\mathrm{X} \text { and the interior of } \mathrm{Y} \text {; There is no } \\
\text { intersection between the interior of } \mathrm{X} \\
\text { and the boundary of } \mathrm{Y} \text {; There is an } \\
\text { intersection between the interiors of } \\
\mathrm{X} \text { and } \mathrm{Y} \text {. }\end{array}$ & & $\begin{array}{l}X \text { and } Y \text { are } \\
\text { equal. }\end{array}$ & Equality \\
\hline
\end{tabular}




\begin{tabular}{|c|c|c|c|c|c|}
\hline$\left(\begin{array}{c}\varnothing \\
\neg \varnothing\end{array}\right.$ & $\left.\begin{array}{c}\varnothing \\
\neg \varnothing\end{array}\right)$ & $\begin{array}{l}\text { There is no intersection between the } \\
\text { boundaries of } \mathrm{X} \text { and } \mathrm{Y} \text {; There is no } \\
\text { intersection between the boundary of } \\
\mathrm{X} \text { and the interior of } \mathrm{Y} \text {; There is an } \\
\text { intersection between the interior of } \mathrm{X} \\
\text { and the boundary of } \mathrm{Y} \text {; There is an } \\
\text { intersection between the interiors of } \\
\mathrm{X} \text { and Y. }\end{array}$ & $X$ & $\begin{array}{l}X \text { contains } Y \text {, } \\
\text { and the } \\
\text { boundaries } \\
\text { of the two do } \\
\text { not intersect. }\end{array}$ & Containing \\
\hline$\left(\begin{array}{l}\varnothing \\
\varnothing\end{array}\right.$ & $\left.\begin{array}{l}\neg \varnothing \\
\neg \varnothing\end{array}\right)$ & $\begin{array}{l}\text { There is no intersection between the } \\
\text { boundaries of } \mathrm{X} \text { and Y; There is an } \\
\text { intersection between the boundary of } \\
\mathrm{X} \text { and the interior of Y; There is no } \\
\text { intersection between the interior of } \mathrm{X} \\
\text { and the boundary of Y; There is an } \\
\text { intersection between the interiors of } \\
\mathrm{X} \text { and Y. }\end{array}$ & $Y$ & $\begin{array}{l}X \text { is } \\
\text { contained in } \\
Y \text { and the } \\
\text { boundaries } \\
\text { of the two do } \\
\text { not intersect. }\end{array}$ & Contained \\
\hline$\left(\begin{array}{l}\neg \varnothing \\
\neg \varnothing\end{array}\right.$ & $\left.\begin{array}{c}\varnothing \\
\neg \varnothing\end{array}\right)$ & $\begin{array}{l}\text { There is an intersection between the } \\
\text { boundaries of } \mathrm{X} \text { and } \mathrm{Y} \text {; There is no } \\
\text { intersection between the boundary of } \\
\mathrm{X} \text { and the interior of } \mathrm{Y} \text {; There is an } \\
\text { intersection between the interior of } \mathrm{X} \\
\text { and the boundary of } \mathrm{Y} \text {; There is an } \\
\text { intersection between the interiors of } \\
\mathrm{X} \text { and Y. }\end{array}$ & $\bar{X}$ & $\begin{array}{l}X \text { covers } Y, \\
\text { and the } \\
\text { boundaries } \\
\text { of both } \\
\text { intersect. }\end{array}$ & Covering \\
\hline$\left(\begin{array}{c}\neg \varnothing \\
\varnothing\end{array}\right.$ & $\left.\begin{array}{l}\neg \varnothing \\
\neg \varnothing\end{array}\right)$ & $\begin{array}{l}\text { There is an intersection between the } \\
\text { boundaries of } \mathrm{X} \text { and Y; There is an } \\
\text { intersection between the boundary of } \\
\mathrm{X} \text { and the interior of Y; There is no } \\
\text { intersection between the interior of } \mathrm{X} \\
\text { and the boundary of Y; There is an } \\
\text { intersection between the interiors of } \\
\mathrm{X} \text { and Y. }\end{array}$ & $Y$ & $\begin{array}{l}X \text { covers } Y \\
\text { and the } \\
\text { boundaries } \\
\text { of the two } \\
\text { intersect }\end{array}$ & Covered \\
\hline$\left(\begin{array}{l}\varnothing \\
\varnothing\end{array}\right.$ & $\left.\begin{array}{l}\varnothing \\
\varnothing\end{array}\right)$ & $\begin{array}{l}\text { There is no intersection between the } \\
\text { boundaries of } \mathrm{X} \text { and } \mathrm{Y} \text {; There is no } \\
\text { intersection between the boundary of } \\
\mathrm{X} \text { and the interior of } \mathrm{Y} \text {; There is no } \\
\text { intersection between the interior of } \mathrm{X} \\
\text { and the boundary of } \mathrm{Y} \text {; There is no } \\
\text { intersection between the interiors of } \\
\mathrm{X} \text { and } \mathrm{Y} \text {. }\end{array}$ & & $\begin{array}{l}X \text { and } Y \text { are } \\
\text { separated } \\
\text { from each } \\
\text { other }\end{array}$ & Separation \\
\hline
\end{tabular}

As shown in Table 1, topological spatial relationships include the following 8 categories: connection, intersection, equality, including, included, covering, covered, and separation.

To sum up, spatial relations include spatial distance relations, spatial direction relations and spatial topological relations. Among them, the spatial topological relation can be divided into 8 subtypes, such as connection, intersection, equality, including, included, covering, covered and separation. Spatial topological relations are "symmetrical" semantic relations because they have the same topological properties under topological changes. However, the spatial distance relation and the spatial direction relation are "asymmetric" semantic relations due to the interference of distance identification and direction identification.

\section{RECiprocal CONDITIONS For SPATIAL MirRor InVERSED SENTENCES}

Spatial mirror inversed sentences only have the subtype of simple spatial relation, such as examples (1)-(4) (all the example have been taken from the previous literatures). This section carefully examines the reciprocal conditions of this type of mirror inversed sentence.

(1) Zhixian A pingxingyu zhixian $B \leftrightarrow$ Zhixian B pingxingyu zhixian A (Tao, 1987)

Line $\mathrm{A}$ is parallel to line $\mathrm{B} \leftrightarrow$ Line $\mathrm{B}$ is parallel to line $\mathrm{A}$

(2) Wuhan kaozhe Changjiang $\leftrightarrow$ Changjiang kaozhe Wuhan. (Bai, 1994)

Wuhan is close to the Yangtze River $\leftrightarrow$ The Yangtze River is close to Wuhan. 
(3) Xiaomen duizhe chezhan $\leftrightarrow$ Chezhan duizhe xiaomen (Zhang and Chen, 2000)

The school gate faces the station $\leftrightarrow$ The station faces the school gate

(4) Youju de zhengmen

chaozhe women de suse $\leftrightarrow$ Women de suse chaozhe youju de zhengmen (Zhang and Chen, 2000)

The main entrance of the post office faces our dormitory $\leftrightarrow$ Our dormitory faces the main entrance of the post office

According to section 2, spatial relations can be divided into three categories: spatial distance relations, spatial direction relations and spatial topological relations. Among them, the spatial topological relation can be divided into 8 sub-types: connection, intersection, equality, including, included, covering, covered, and separation. After investigation, it is found that the mirror inversed expressions of examples (1)-(4) belong to spatial topological relations. Among them, examples (1), (3) and (4) belong to the topological relation of spatial separation, and example (2) belongs to the topological relation of spatial connection.

Let's take the example (1) "Zhixian A pingxingyu zhixian B (Line A is parallel to line B) Zhixian B pingxingyu zhixian A (Line B is parallel to line A)" as an example to explain the reciprocity conditions of mirror inversed sentences with spatial separation.

In Example (1), "Line A" and "Line B" are both lines, and the two have a line-line spatial relationship as shown in Fig. 1:

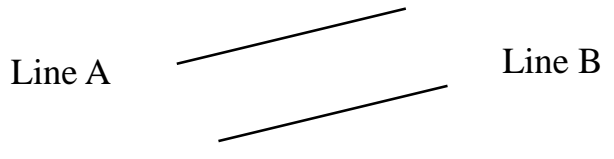

Fig1. The line-line spatial relationship of example (1)

According to Fig. 1, There is no intersection between the boundaries of line A and line B; There is no intersection between the boundary of line $\mathrm{A}$ and the interior of line $\mathrm{B}$; There is no intersection between the interior of line A and the boundary of line B; There is no intersection between the interiors of line A and line B.These characteristics are consistent with the definition of spatial separation (see Table 1 for details); As such, "line A" and "line B" have a topological relationship of spatial separation. In other words, example (1) belongs to the mirror inversed sentence of spatial topological relation.

Next, we will try to change the spatial topological relationship between the body words "line A" and "line B" in example (1), and verify the reciprocity of the body words under non-spatial topological relationship (i.e. spatial distance relationship and spatial direction relationship) and the possibility of forming mirror inversed sentences. The specific verification results are shown in Table 2:

Table2. Test of sentence pattern variants and body words reciprocity for example (1)

\begin{tabular}{|l|l|l|l|l|}
\hline $\begin{array}{l}\text { Changed } \\
\text { relationship }\end{array}$ & Syntactic operation & $\begin{array}{l}\text { Sentences before } \\
\text { reciprocity }\end{array}$ & $\begin{array}{l}\text { Sentences after } \\
\text { reciprocity }\end{array}$ & MISs? \\
\hline $\begin{array}{l}\text { Spatial } \\
\text { distance } \\
\text { relation }\end{array}$ & $\begin{array}{l}\text { Delete "pingxingyu"; Add the } \\
\text { distance marker "li... buyuan" }\end{array}$ & $\begin{array}{l}\text { Zhixian A li zhixian } \\
\text { B buyuan. (Line A is } \\
\text { not far from line B.) }\end{array}$ & $\begin{array}{l}\text { Zhixian B li zhixian A } \\
\text { buyuan. (Line B is not } \\
\text { far from line A.) }\end{array}$ & $\begin{array}{l}\text { Reciprocal } \\
\text { but } \\
\text { MIS }\end{array}$ \\
\hline $\begin{array}{l}\text { Spatial } \\
\text { direction } \\
\text { relation }\end{array}$ & $\begin{array}{l}\text { Delete "pingxingyu"; Add the } \\
\text { direction marker "zai... } \\
\text { xiamian" }\end{array}$ & $\begin{array}{l}\text { Zhixian A zai zhixian } \\
\text { B xiamian. (Line A is } \\
\text { under line B.) }\end{array}$ & $\begin{array}{l}\text { Zhixian B zai zhixian } \\
\text { A xiamian. (Line B is } \\
\text { under line A.) }\end{array}$ & $\begin{array}{l}\text { Reciprocal } \\
\text { but } \\
\text { MIS }\end{array}$ \\
\hline
\end{tabular}

As shown in Table 2, when we change the spatial topological relation of example (1) to the spatial distance relation and the spatial direction relation, the changed setence can realize the reciprocity of body words but cannot form mirror inversed sentences. In this way, the necessary condition for the mirror inverse sentence similar to (1) to realize the reciprocity of body words to become the mirror inversed sentence is that the mirror inversed sentence patterns before and after reciprocity all have the spatial topological relation.

Then, taking the example (2) "Wuhan kaozhe Changjiang (Wuhan is close to the Yangtze River) $\leftrightarrow$ 
Changjiang kaozhe Wuhan (The Yangtze River is close to the Yangtze River)" as an example, the reciprocal conditions of mirror inversed sentences with spatial connection topological relations are explained.

In Example (2), if "the Yangtze River" is regarded as a line and "Wuhan"is regarded as a surface, the two have a line-surface spatial relationship as shown in Figure 2:

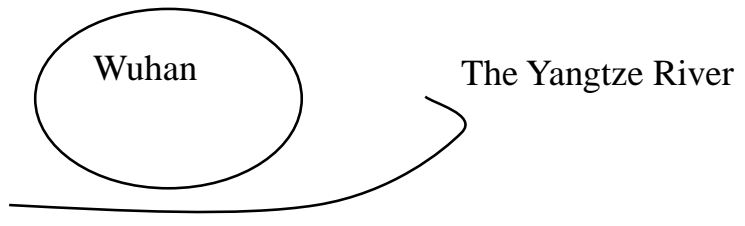

Fig2. The line-surface spatial relationship of example (2)

According to Fig. 2, There is an intersection between the boundaries of Wuhan and the Yangtze River; There is no intersection between the boundary of Wuhan and the interior of the Yangtze River; There is no intersection between the interior of Wuhan and the boundary of the Yangtze River; There is no intersection between the interiors of Wuhan and the Yangtze River. These characteristics are consistent with the definition of spatial connction (see Table 1 for details); As such, "Wuhan" and "the Yangtze River" have a topological relationship of spatial connection. In other words, example (2) belongs to the mirror inversed sentence of spatial topological relation.

Next, we will try to change the spatial topological relationship between the body words "Wuhan" and "the Yangtze River" in example (2), and verify the reciprocity of the body words under non-spatial topological relationship (i.e. spatial distance relationship and spatial direction relationship) and the possibility of forming mirror inversed sentences. The specific verification results are shown in Table 3:

Table3. Test of sentence pattern variants and body words reciprocity for example (2)

\begin{tabular}{|l|l|l|l|l|}
\hline $\begin{array}{l}\text { Changed } \\
\text { relationship }\end{array}$ & Syntactic operation & $\begin{array}{l}\text { Sentences before } \\
\text { reciprocity }\end{array}$ & $\begin{array}{l}\text { Sentences after } \\
\text { reciprocity }\end{array}$ & MISs? \\
\hline $\begin{array}{l}\text { Spatial } \\
\text { distance } \\
\text { relation }\end{array}$ & $\begin{array}{l}\text { Delete "kaozhe"; Add the } \\
\text { distance marker "zai... } \\
\text { pangbian" }\end{array}$ & $\begin{array}{l}\text { Wuhan } \\
\text { Changjiang } \\
\text { pangbian (Wuhan is } \\
\text { next to the Yangtze } \\
\text { River) }\end{array}$ & $\begin{array}{l}\text { Changjiang } \\
\text { Wuhan pangbian } \\
\text { the Yangtze River } \\
\text { is next to Wuhan) }\end{array}$ & $\begin{array}{l}\text { Reciprocal but } \\
\text { not MIS }\end{array}$ \\
\hline $\begin{array}{l}\text { Spatial } \\
\text { direction } \\
\text { relation }\end{array}$ & $\begin{array}{l}\text { Delete "kaozhe"; Add the } \\
\text { distance marker "zai... } \\
\text { xiayou" }\end{array}$ & $\begin{array}{l}\text { Wuhan } \\
\text { Changjiang xiayou } \\
\text { (Wuhan is in the } \\
\text { lower reaches of the } \\
\text { Yangtze River) }\end{array}$ & $\begin{array}{l}\text { Changiang } \\
\text { Yuhan xiayou (the } \\
\text { the lower reaches of } \\
\text { Wuhan) }\end{array}$ & No \\
\hline
\end{tabular}

As shown in Table 3, when we change the spatial topological relation of example (2) to the spatial distance relation, the changed example can realize the reciprocity of body words but cannot form mirror sentences. When the spatial topological relation of the example is changed to the spatial direction relation, the changed example cannot realize the reciprocity of body words to form mirror inversed sentences. In this way, the necessary condition for the mirror inversed sentence similar to (2) to realize the reciprocity of body words to become the mirror inverse sentence is that the mirror inverse sentence patterns before and after reciprocity all have the spatial topological relation.

Through the investigation of other examples of the spatial MISs, it is found that the necessary conditions for simple spatial relation MISs to realize the reciprocity of body words to become MISs are: the MISs before and after reciprocity have spatial topological relation. Since the spatial topological relation is the same topological property under topological transformation, describing the topological positional relation between geometric objects is a "symmetrical" semantic relation. It can be inferred from this that the existence of "symmetrical" semantic relation is a necessary condition for the simple spatial relation mirror inverse sentence to realize the reciprocity of body words. 


\section{CONCLUSION}

From the perspective of spatial relations, this paper focuses on the specific conditions for the reciprocity of body words in the spatial mirror inversed sentence pattern.

Firstly, this paper discusses the specific classification of spatial relations. Spatial relations can be generally discussed from three angles: spatial distance relations, spatial direction relations and spatial topological relations. Spatial topological relations can be divided into 8 sub-relations according to the topological definition of point sets: connection, intersection, equality, including, included, covering, covered and separation.

Then, this paper focuses on the specific conditions for the reciprocity of body words in the spatial mirror inversed sentence pattern. The necessary condition for the reciprocity of the body words in the spatial mirror inversed sentence is the "symmetrical" spatial topological relationship between the two body words. In short, the existence of "symmetrical" semantic relations in the spatial dimension is a common necessary condition for the reciprocity of body words in spatial mirror inversed sentences. In other words, the symmetry of the conceptual semantics of the mirror inversed sentence pattern is the premise of its formal symmetry, and the formal symmetry is the reflection of its conceptual semantic symmetry. This is related to the iconicity of human cognition, specifically, to the iconicity motivation of human symmetry.

\section{ACKNOWLEDGEMENT}

The research was supported by the Scientific Research Fund of Sichuan University of Science and Engineering (B40101351)

\section{REFERENCES}

[1] Bai D., On Chinese Double-faced Verbs, Journal of South-Central University for Nationalities (Humanities and Social Sciences), No. 5, pp. 115-120, (1994).

[2] Chen J. M., On Sentence Patterns in Modern Chinese, Beijing: Chinese publishing house, (1986).

[3] Chen P., On the Coordination Principle of Three Sentence Components and Semantic Components in Chinese, Studies of the Chinese Language, No. 3, pp. 161-168, (1994).

[4] Ding et al., Modern Chinese Grammar Speech (Edition 1), Beijing: The Commercial Press, (1961).

[5] Ding J. Y., Quantitative Relationship, Syntactic Features and Cognitive Interpretation of Accommodation Sentences, Chinese Linguistics, No. 1, pp. 64-75+95, (2006).

[6] Gan X. L., The Attribute-space-time Classification of Mirror-Image Inversion Sentence in Modern Chinese, International Journal of Education Humanities and Social Science, No. 5, pp. 94-106, (2019).

[7] Her O.S., Apparent subject-object inversion in Chinese, Linguistics, No. 5, pp. 1143-1181, (2009).

[8] Huang C.R., Mandarin Chinese and the Lexical Mapping Theory: a Study of the Interaction of Morphology and Argument Changing, Journal of Institute of History and Language, Academia Sinica, No. 1, pp. 337-388, (1993).

[9] Kant I., Critique of Pure Reason, Deng X. M. (Ed), Beijing: People's Publishing House, (2004).

[10] Li L. D., Which one "complements" which one? Re-discussion on the relationship of "verb-complement", Chinese Learning, No. 2, pp. 1-10, (1984).

[11] Lu J. M., "Sentence Grammar" Theory and Chinese Studies, Studies of the Chinese Language, No. 5, pp. 412-416+479, (2004).

[12] Lv S. X., Recent Writings about Chinese, Shanghai: Shanghai Education Press, (1987).

[13] Ren Y., An Analysis of Predicate Structure of Subject-object Transposable Verb-construction, Studies of the Chinese Language, No. 4, pp. 320-328+384, (2001).

[14] Tao H. Y., Mutual Verbs and Mutual Verb Sentences, Symposium on Sentence Patterns and Verbs, (1987).

[15] Wang X. J., Syntactic polysemy of "Xiang" verbs, Chinese Learning, No. 2, pp. 7-13, (1992).

[16] Yue Y., On the Semantic Origin of "S+W+ si $+\mathrm{O}$ " Sentence Pattern's Causative and Automatic Meaning, Journal of Tsinghua University (Philosophy and Social Sciences Edition), No. S2, pp. 134-142+144. (2009).

[17] Zhang B and Chen C. L., Modern Chinese Sentences, Shanghai: East China Normal University Press, (2000).

[18] Zhang R. F., On the Expression of Verbal Sentence Phrases, Studies of the Chinese Language, No. 2, pp. 105-111. (1989). 
[19] Zhu D. X., "Zai heiban shang xie zi" and Related Sentences, Language Teaching and Linguistic Studies, No. 1, pp. 4-18. (1981).

\section{AUTHOR'S BIOGRAPHIES}

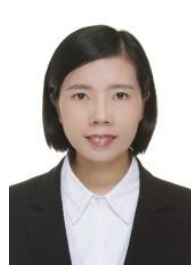

Xiaolan Gan, PhD. Associate Professor of Linguistics Sichuan University of Science and Engineering Zigong, Si Chuan, 643000

Citation: Xiaolan Gan. "Reciprocal Conditions for Spatial Mirror Inversed Sentences in Modern Chinese" International Journal of Humanities Social Sciences and Education (IJHSSE), vol 7, no. 10, 2020, pp. 69-77. doi: https://doi.org/10.20431/2349-0381.0710009.

Copyright: (c) 2020 Authors. This is an open-access article distributed under the terms of the Creative Commons Attribution License, which permits unrestricted use, distribution, and reproduction in any medium, provided the original author and source are credited. 